\title{
BMJ Open Meta-analysis of the diagnostic value of Wisteria floribunda agglutinin-sialylated mucin1 and the prognostic role of mucin1 in human cholangiocarcinoma
}

\author{
Zengwei Tang, ${ }^{1}$ Yuan Yang, ${ }^{1}$ Xiaolu Wang, ${ }^{2}$ Wenbo Meng, ${ }^{1,3}$ Xun $\mathrm{Li}^{1,4}$
}

To cite: Tang Z, Yang $Y$, Wang $X$, et al. Meta-analysis of the diagnostic value of Wisteria floribunda agglutinin-sialylated mucin1 and the prognostic role of mucin1 in human cholangiocarcinoma. BMJ Open 2019;9:e021693. doi:10.1136/ bmjopen-2018-021693

- Prepublication history and additional material for this paper are available online. To view these files, please visit the journal online (http://dx.doi. org/10.1136/bmjopen-2018021693).

Received 12 January 2018 Revised 10 November 2018 Accepted 13 November 2018

\section{Check for updates}

(c) Author(s) (or their employer(s)) 2019. Re-use permitted under CC BY-NC. No commercial re-use. See rights and permissions. Published by BMJ.

${ }^{1}$ The First Clinical Medical School of Lanzhou University, Lanzhou, China

${ }^{2}$ Department of General Surgery, West China Hospital/West China Medical School, Sichuan University, Chengdu, China ${ }^{3}$ Department of Special Minimally Invasive Surgery, The First Hospital of Lanzhou University, Lanzhou, Gansu Province, China

${ }^{4}$ The Second Department of General Surgery, The First Hospital of Lanzhou University, Lanzhou, China

Correspondence to

Dr Wenbo Meng;

mengwb@|zu.edu.cn

\section{ABSTRACT}

Objective Serum carbohydrate antigen 19-9 (CA19-9) is a widely used tumour marker for cholangiocarcinoma (CCA). However, it is not a necessarily good CCA marker in terms of diagnostic accuracy. The purpose of this study is to evaluate the diagnostic value of Wisteria floribundaagglutinin-sialylated Mucin1 (WFA-MUC1) and the prognostic role of Mucin1 (MUC1) in human CCA.

Design Meta-analysis.

Data sources Studies published in PubMed, Web of Science, The Cochrane Library and the China National Knowledge Infrastructure up to 11 October 2017. Eligibility criteria We included reports assessing the diagnostic capacity of WFA-MUC1 and the prognostic role of MUC1 in CCA. The receiver operating characteristic curve (ROC) of WFA-MUC1 and/or CA19-9 was described, and the HRs including $95 \% \mathrm{Cl}$ and the corresponding $p$ value for MUC1 can be extracted.

Data extraction and synthesis Two independent researchers extracted data and assessed risk of bias. The diagnostic sensitivity and specificity data of WFA-MUC1 were extracted and analysed as bivariate data. Pooled HRs and its $95 \% \mathrm{Cl}$ for MUC1 were calculated with a random-effects meta-analysis model on overall survival of resectable CCA.

Results Sixteen reports were included in this study. The pooled sensitivity and specificity of WFA-MUC1 were $0.76(95 \% \mathrm{Cl} 0.71$ to 0.81$)$ and $0.72(95 \% \mathrm{Cl}$ 0.59 to 0.83$)$ in serum, $0.85(95 \% \mathrm{Cl} 0.81$ to 0.89$)$ and $0.72(95 \% \mathrm{Cl} 0.64$ to 0.80$)$ in bile and $0.72(95 \% \mathrm{Cl}$ 0.50 to 0.87$)$ and 0.85 (95\% $\mathrm{Cl} 0.70$ to 0.93$)$ in tissue, respectively. The summary ROC (SROC) were 0.77 $(95 \% \mathrm{Cl} 0.73$ to 0.81$)$ in serum, 0.88 (95\% Cl 0.85 to $0.90)$ in bile and $0.86(95 \% \mathrm{Cl} 0.83$ to 0.89$)$ in tissue, respectively. Furthermore, the pooled sensitivity and specificity and the SROC of CA19-9 in serum were 0.67 (95\% Cl 0.61 to 0.72$), 0.86$ (95\% Cl 0.75 to 0.93 ) and 0.75 (95\% Cl 0.71 to 0.79$)$, respectively. The pooled HRs for MUC1 was 2.20 (95\% Cl 1.57 to 3.01) in CCA and 4.17 (95\% Cl 1.71 to 10.17$)$ in mass-forming intrahepatic CCA.

Conclusions Compared with CA19-9, WFA-MUC1 was shown to possess stronger diagnostic capability. MUC1 could serve as a prognosis factor for poor outcomes of CCA, particularly, mass-forming intrahepatic CCA.
Strengths and limitations of this study

- This meta-analysis evaluated the diagnostic capability of Wisteria floribunda agglutinin-sialylated Mucin1 (WFA-MUC1) and prognostic role of Mucin1 (MUC1) in cholangiocarcinoma.

- The diagnostic capability of WFA-MUC1 is superior to that of CA19-9.

- The diagnostic capability of WFA-MUC1 in bile is better that in serum.

- Expression of MUC1 in biliary duct cancer tissues is a prognosis factor for poor outcomes of resectable cholangiocarcinoma.

- Majority of the subjects included in this meta-analysis were from Asia. More participants from different regions other than Asia are needed to better evaluate the roles of Mucin1 in the diagnosis and prognosis of cholangiocarcinoma worldwide.

\section{INTRODUCTION}

Cholangiocarcinoma (CCA) is a malignancy arising from epithelia at various anatomic locations in the biliary tree. ${ }^{1}$ The median survival time for patients with unresectable CCA is less than a year. ${ }^{23}$ The prognosis is considerably better for CCA patients who underwent radical resection, with a 5-year survival rates ranging from $20 \%$ to $40 \% .{ }^{45}$ However, it is hard to detect CCA at the early stage, even with the advanced imaging technology and the complete diagnosis protocol currently. This situation limits the benefits of surgery therapy and curative treatment options to CCA patients and contributes to the poor outcome of patients with CCA.

Currently, a huge amount of literature reporting numerous molecular biomarkers with limited diagnostic or prognostic capability for CCA have been published. Some of the reported biomarkers have been used for guiding clinical diagnosis and treatment of CCA worldwide, such as Mucin2 to Mucin6, ${ }^{6-15}$ carbohydrate antigen 19-9 (CA19$9),{ }^{16-18}$ interleukin-6, ${ }^{19}{ }^{20}$ serum cytokeratin 19 
fragments $^{2122}$ and carbohydrate antigen 125. ${ }^{162324}$ Among these biomarkers, CA19-9 in serum has been the focus of related research and always been used as a biomarker for CCA. However, the overall sensitivity and specificity of CA19-9 is not satisfying, and CA19-9 is not capable of detecting CCA progression. ${ }^{5} 1724$ In addition, although CA19-9 expression is elevated in up to $85 \%$ suspected $\mathrm{CCA},{ }^{1725} 26$ the capability of CA19-9 as a diagnostic marker is still limited due to influence of coexisting inflammation in biliary tract and the fact that cancer cells from Lewis gene negative subtype of CCA does not produce CA19-9 theoretically. 171827

Mucin1 (MUC1), also known as polymorphic epithelial Mucin, is cell surface associated and belongs to Mucin family. It is a mucin encoded by the MUC1 gene in humans. ${ }^{28}$ MUC1 is a high molecular weight, membrane-associated glycoprotein with a 69 amino acids cytoplasmic tail, a transmembrane domain and an extracellular domain consisting of a variable number of highly conserved tandem repeats of 20 amino acids. $^{28}{ }^{29}$ Highly glycosylated MUC1 has been reported to be associated with malignancies in many other organs. ${ }^{30}$ Matsuda $e t a l^{11}$ reported that Wisteria floribunda agglutinin-sialylation (WFA) could be employed as the best probe to detect alterations of glycan structure in biliary tract-derived cancer cells and distinguish it from normal tissues. They also identified sialylated MUC1 as a potential CCA-specific glycoprotein marker. From then on, Wisteria floribunda agglutinin sialylated-Mucin1 (WFA-MUC1) has been regarded as a sensitive molecular biomarker for CCA. ${ }^{931-35}$ However, the diagnostic capability of WFA-MUC1 remains unclear since the reported range of WFA-MUC1 distinguishing CCA from benign biliary diseases varied greatly $(0.74 \sim 0.87$ in serum, $0.72 \sim 0.90$ in bile)..$^{93-35}$ In addition, although the correlation between the expression of MUC1 in biliary duct-derived cancer and the overall survival (OS) rate for patients with resectable CCA has been analysed with Kaplan-Meier plot in several clinical trials, the result still remains inconclusive. Besides, more questions about MUC1 in CCA still need to be answered such as whether expression of MUC1 suggests a poor prognosis for patients with CCA and whether expression level of MUC1 associates with CCA progression. ${ }^{79} 1012-153637$

Therefore, we conducted this meta-analysis to evaluate the diagnostic capability of WFA-MUC1 in discriminating patients with CCA from benign biliary diseases and to investigate the prognostic role of MUC1 in patients with CCA.

\section{METHODS}

\section{Search strategy}

The initial comprehensive literature search through 11 March 2017 was performed in database of PubMed, Web of Science, The Cochrane Library and the China National Knowledge Infrastructure. Our latest search was completed on 11 October 2017. The publication language was restricted to articles published in English or Chinese. Searching keywords used are 'Wisteria floribunda agglutinin sialylated-mucin1(WFA-MUC1)', 'Mucin1/MUC1',
'cholangiocarcinoma/CCA', 'cholangiocellular carcinoma', 'intrahepatic cholangiocarcinoma', 'extrahepatic cholangiocarcinoma' or 'Klatskin tumor/hilar cholangiocarcinoma/perihilar cholangiocarcinoma' combined with 'prognosis/prognostic/prognoses/survival' or 'diagnosis/diagnostic/diagnoses'. The reference lists of every study that met the inclusion criteria were also manually reviewed to identify additional relevant publications.

\section{Patient and public involvement}

Patients and public were not involved as all the data used have been published previously and hence are already in the public domain.

\section{Eligibility criteria}

Published studies were included if they met the following criteria: (1) the published studies were focused on CCA; (2) all studied subjects with CCA were diagnosed by pathologist postoperatively; (3) the expression of MUC1 in tissues was detected by immunohistochemistry staining and the level of WFA-MUC1 in bile or serum was tested by sandwich ELISA; (4) the receiver operating characteristic curve (ROC) of WFA-MUC1 and/or CA19-9 was described and the rates of true positives, false positives, false negatives and true negatives can be calculated; and (5) the HRs including 95\% CI and the corresponding $\mathrm{p}$ value can be extracted. Studies were excluded based on following criteria: (1) animal studies; (2) review articles, case reports or letters; (3) duplicated publication; (4) non-English or non-Chinese papers; and (5) insufficient data on the HRs or that could not be extracted from Kaplan-Meier analysis result.

\section{Data extraction}

Data extraction was carried out by two investigators independently (ZT and $\mathrm{YY}$ ). If discrepancies occurred, it would be resolved by the consensus of these two investigators. Data related to the study characteristics were extracted with the following variables: the first author of the study, study design and duration, year of publication, institution, the number of subjects in the study with mean age and gender, the selected antibody for the MUC1 immunochemical staining, ELISA assay kits testing the level of biliary and/or serum WFA-MUC1 and the level of serum CA-19-9, the area under the curve (AUC) for WFA-MUC1, the cut-off value of MUC1, assay's sensitivity and specificity, HRs and their $95 \%$ CI and case follow-up time. For the three studies that did not provide the value of HRs and their 95\% CI, we digitised and extracted the data from the Kaplan-Meier curve in the publications by using the software designed by Jayne F Tierney and Matthew R Sydes. ${ }^{38}$ The optimal sensitivity and specificity were reported graphically in one study with two cohorts and were extracted using Plot Digitizer software V.2.6.8 (provided by source forge.net, found online at http:// plot digitizer source forge.net/) to convert data points on the graphs into numerical data. ${ }^{39} 40$ Repeated data points were isolated using non-parametric bootstrap 
sampling $^{41}$ guided by the descriptive statistics provided in the supporting document. The possible repeated data points were repeatedly sampled until the sets matching the descriptive statistics was found. All the data were extracted from published literature.

\section{Quality assessment across studies}

Quality assessment of the studies in the prognostic meta-analysis was performed by using the modified risk of bias tool recommended by the Cochrane Collaboration as described previously. ${ }^{42-44}$ Quality assessment of studies evaluating the diagnostic capability of WFA-MUC1 was performed using the Quality Assessment of Studies of Diagnostic Accuracy included in Systematic reviews (QUADAS) checklists. ${ }^{45}{ }^{46}$ However, we did not calculate the summary scores for each study investigating the diagnostic capability of WFA-MUC1 because their interpretation was problematic and because their report was potentially misleading. ${ }^{47}$ Moreover, seven of the best differentiating items have been selected from the QUADAS checklists.

\section{Statistical analyses}

The statistical analysis was performed according to the guidelines proposed by the Meta-Analysis of Observational Studies in Epidemiology group. ${ }^{48}$ The pooled HRs with $95 \%$ CI were calculated with a random-effect model according to the DerSimonian-Laird method to evaluate the correlation between the positive expression of MUC1 and OS. ${ }^{49}{ }^{50}$ Sensitivity and specificity for each study evaluating the diagnostic capability of WFA-MUC1 were calculated and analysed this datum as bivariate data according to methods for diagnostic meta-analysis. ${ }^{51} \mathrm{An}$ aggregated bivariate data meta-analysis with the generation of forest plots and summary ROC (SROC) was performed. Forest plots displayed the diagnostic probabilities of individual studies, the corresponding 95\% CI and squares with area proportional to study weight in the meta-analysis. The SROC demonstrated individual study data point with circles, with size proportion to study weight and $95 \%$ prediction contour and $95 \%$ confidence contour around the pooled estimate. The heterogeneity among studies was measured using the $Q$ tests and $\mathrm{I}^{2}$ statistic to assess the extent of the inconsistency. A probability value of $\mathrm{p}<0.1$ and $\mathrm{I}^{2}>50 \%$ indicated the existence of significant heterogeneity. ${ }^{52}$ Furthermore, funnel plot and the Egger's linear regression test were applied to evaluate potential publication bias for eligible studies using OS as an endpoint. ${ }^{53} \mathrm{~A} p<0.1$ for Egger's test was considered statistically significant. All statistical analyses were performed with Stata/MP V.14.0.

\section{RESULTS}

\section{Study selection}

The study includes results of electronic searches up to 11 October 2017. A total of 341 papers were identified, of which 148 were retrieved for full-text review. Among these
148 publications, 16 studies $^{6} 791012-15313234-375455$ were eligible for the meta-analyses according to the inclusion and exclusion criteria. Nine studies ${ }^{791012-153637}$ out of 16 studies used OS as endpoint, and eight studies ${ }^{69313234355455}$ used the sensitivity and specificity rate as the endpoint (one study reported by Huang et al also provided the data on diagnostic value of MUC1 in tissue). The detailed literature searching process was shown in figure 1 .

\section{Characteristics of the included studies and participants}

Characteristics of eligible studies and their participants were listed in tables 1 , table 2 and table 3 . A total of nine studies ${ }^{791012-153637}$ evaluating the prognostic value of MUC1 for resectable CCA were conducted in four countries (Korea, Japan, China and Thailand), the other seven studies $^{6} 313234355455$ investigating the diagnostic capability of WFA-MUC1 were undertaken in five countries (Japan, UK, Brazil, Thailand and China). Retrospective study design was applied to perform the meta-analysis of prognostic value by all selected studies. The seven studies investigating the diagnostic capability of WFA-MUC1, meaning that discriminating CCA from benign biliary diseases, used prospective study design. All CCA diagnosis included in this study were based on histopathology as reported in the include publications. The sample size of eligible studies evaluating the prognostic value of MUC1 varied greatly, ranging from 27 to 87 with a median size of 56. The sample size of studies investigating the diagnostic capability of WFA-MUC1 ranged from 30 to 303 (median=80) and from 20 to 287 (median=69) for biliary tract carcinoma group and benign biliary diseases group, respectively.

The level of WFA-MUC1 in bile and serum were tested by the approach of ELISA using mAb WFAMY.1E12. The concentration of serum CA19-9 was tested by CA19-9 ELISA kits. The sensitivity, specificity and AUC of each study included in the diagnostic meta-analysis were shown in table 2.

Three studies ${ }^{9} 14{ }^{36}$ investigating the prognostic value of MUC1 for CCA provided the Kaplan-Meier curve, and we digitised and extracted the data of HRs including their corresponding 95\% CI from the curve by using the methods described above. The cut-off value to define positive expression of MUC1 (two trials ${ }^{12}{ }^{37}:>25 \%$, one trial $^{14}:>20 \%$, two trials ${ }^{910}:>10 \%$ and four trials ${ }^{713}{ }^{14}{ }^{36}$ : $>5 \%$ ), the follow-up time (seven trials ${ }^{7} 1013-153637:>50$ months, one trial ${ }^{9}:>20$ months and another one $e^{12}:>15$ months) and the antibody of MUC1 were selected for immunochemistry (mAb DF3, Clone Mab DF3, Clone Ma695, Clone Ma689 and mAb HMPV) were inconsistent (as shown in table 3).

Primary endpoint: the outcomes of diagnostic meta-analysis Three trials ${ }^{35} 55$ including 414 cases of biliary tract carcinoma (59 gall bladder carcinomas and 355 CCA) and 405 subjects with benign biliary diseases investigated the diagnostic capability of WFA-MUC1 level in serum. Figure 2A presented the diagnostic parameters 
Papers identified through searches of PubMed, The Cochran Library, Web of Science and China National Knowledge Infrastructure ( $n=342$ )

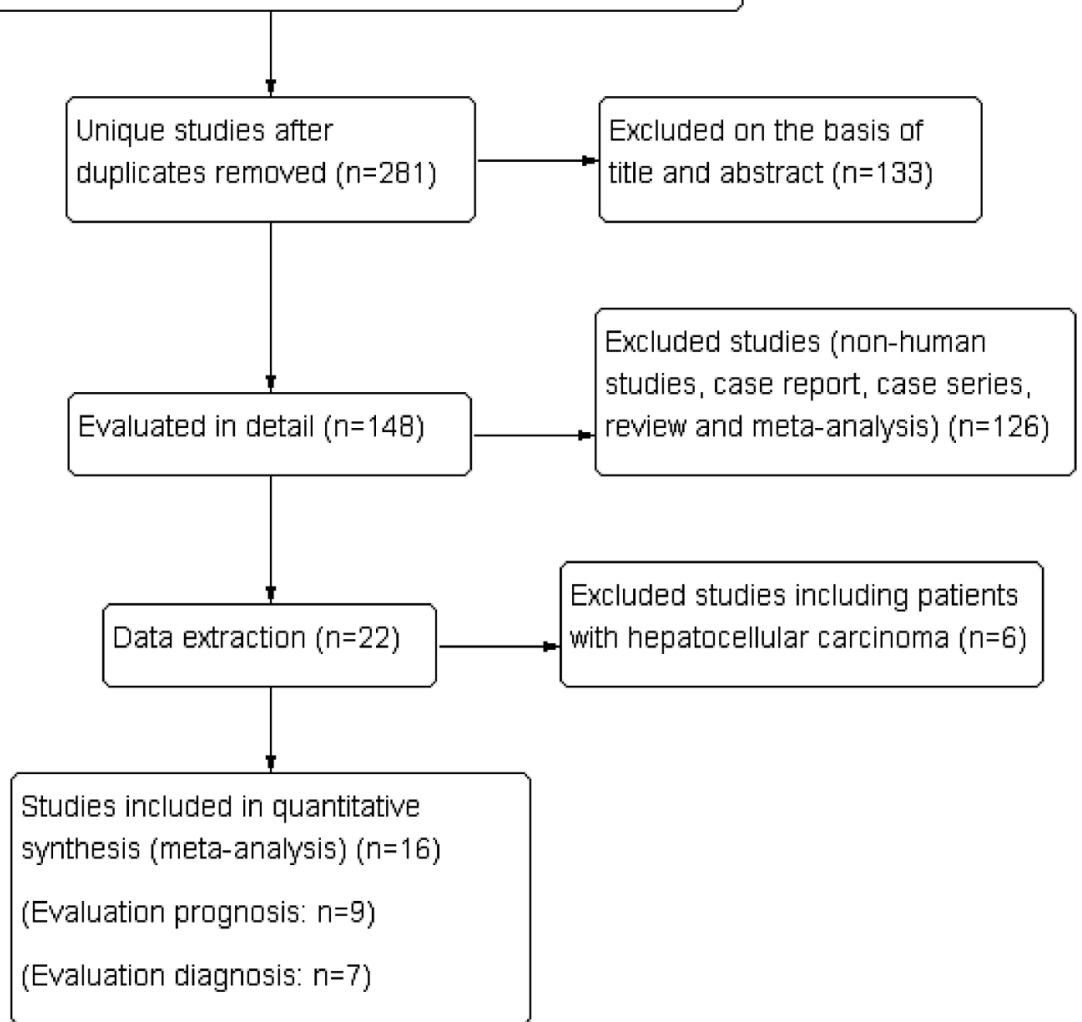

Figure 1 Diagram showing the literature searching work flow.

$\mathrm{s}$ for serum WFA-MUC1 in an SROC graph. The pooled optimal sensitivity (true positive rate) was 0.76 (95\% CI 0.71 to 0.81 ) and specificity (true negative rate) was 0.72 (95\% CI 0.59 to 0.83$)$. The AUC of SROC was $0.77(95 \%$ CI 0.73 to 0.81 ).

As a comparison, three trials ${ }^{34} 3555$ with 588 subjects with biliary tract carcinoma (73 subjects with gall bladder carcinoma and 515 CCA) and 432 subjects with benign biliary disease evaluated the diagnostic capability of CA19-9 level in serum. Figure 2B presented the diagnostic parameters for serum level of CA19-9 in a SROC graph. The pooled optimal sensitivity was 0.67 (95\% CI 0.61 to 0.72 ) and specificity was 0.86 (95\% CI 0.75 to 0.93$)$. The AUC under SROC was 0.75 (95\% CI 0.71 to 0.79 ).

Four trials $^{3132} 3435$ including 209 subjects with benign biliary disease and 416 biliary tract carcinomas (73 gall bladder carcinomas) evaluated the diagnostic capability of biliary level of WFA-MUC1. SROC of biliary WFA-MUC1 was shown in figure 2C. The pooled sensitivity was 0.85 (95\% CI 0.81 to 0.89 ) and specificity was 0.72 (95\% CI 0.64 to 0.80$)$. The AUC under SROC was 0.88 (95\% CI 0.85 to 0.90 ). Furthermore, three trials ${ }^{694}$ inculding 72 subjects with CCA and 119 benign biliary disease used the positive expression of MUC1 in tissue as a criterium to discriminate CCA from benign biliary disease. The diagnostic parameters of positive expression of MUC1 in biliary duct cancer tissue were shown in figure 2D. The pooled sensitivity was 0.72 (95\% CI 0.50 to 0.87 ) and specificity 0.85 (95\% CI 0.70 to 0.93 ). The AUC of SROC was 0.86 (95\% CI 0.83 to 0.89$)$.

Secondary endpoint: the outcome of prognostic meta-analysis Nine studies ${ }^{791012-153637}$ with a total of 511 individuals diagnosed with CCA were eligible for the pooled analysis of OS. As shown in the figure 3, the overall pooled HRs of MUC1 was 2.20 (95\% CI 1.57 to 3.01). No heterogeneity among these studies was found $\left(\mathrm{I}^{2}=0 ; \mathrm{p}=0.869\right)$. Subgroup analyses stratified by the histopathological morphology of CCA reveal that the pooled HRs of mass-forming intrahepatic CCA was 4.17 (95\% CI 1.71 to 10.17). The pooled HRs of CCA was 1.98 (95\% CI 1.37 to 2.85).

\section{Risk of bias within studies}

Detailed results of the risk of bias assessment for included studies in prognostic meta-analysis were shown in online supplementary table 1 . As shown in supplementary table 1 , one study ${ }^{12}$ that showed a high risk of bias, six showed s $^{10131537}$ a low risk of bias and two ${ }^{1436}$ were shown with the unclear risk of bias. Moreover, as demonstrated in figure 4, the result of funnel plots of OS showed no clear indication of publication bias (Egger's test, $\mathrm{p}=0.661$ ). Selection bias of diagnostic analyses may be caused by two trials including 73 subjects diagnosed with gall bladder carcinoma. ${ }^{34} 35$ Detailed items selected for quality assessment of studies included in diagnostic meta-analysis was shown in online supplementary table 2 . 


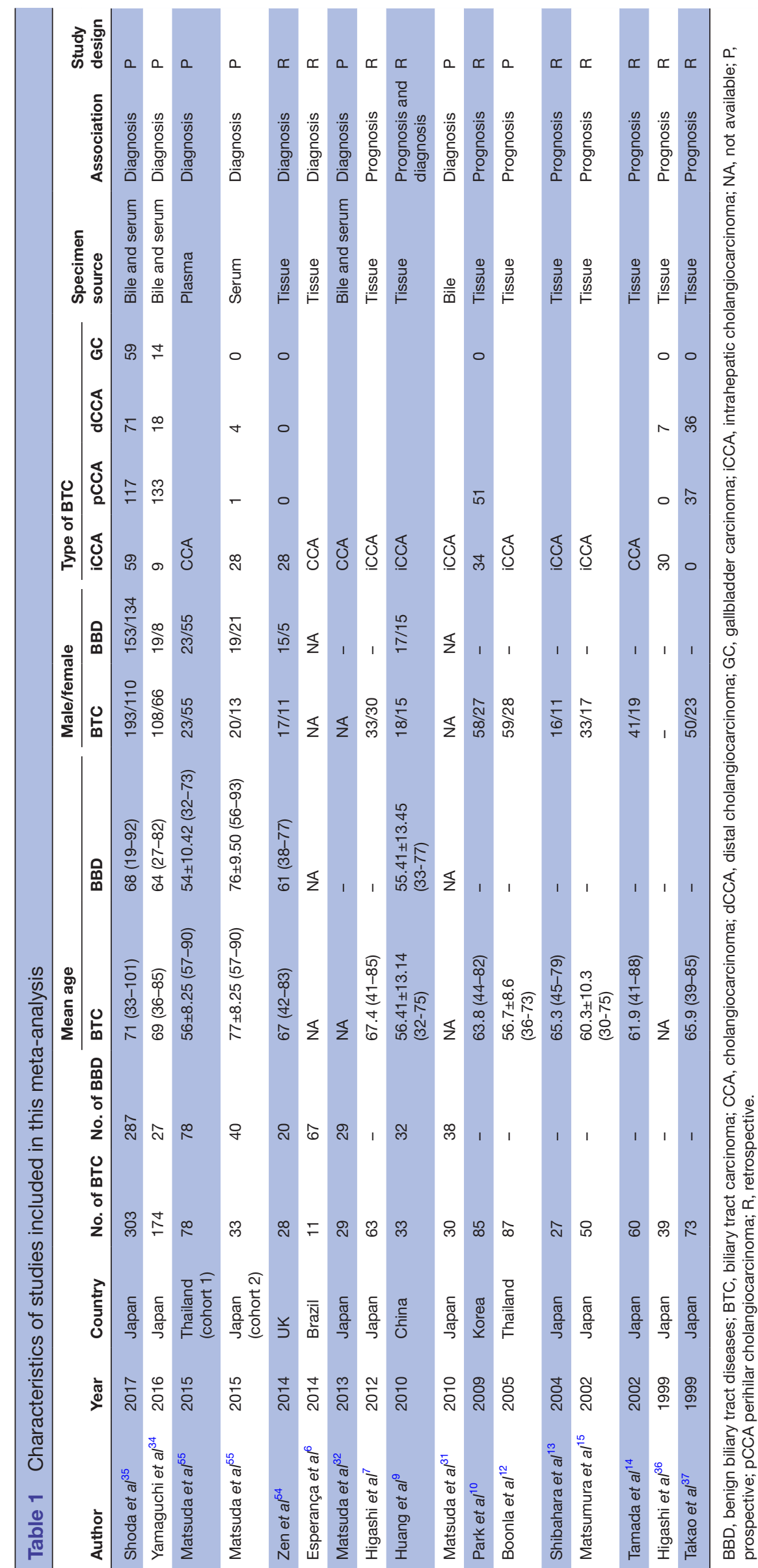




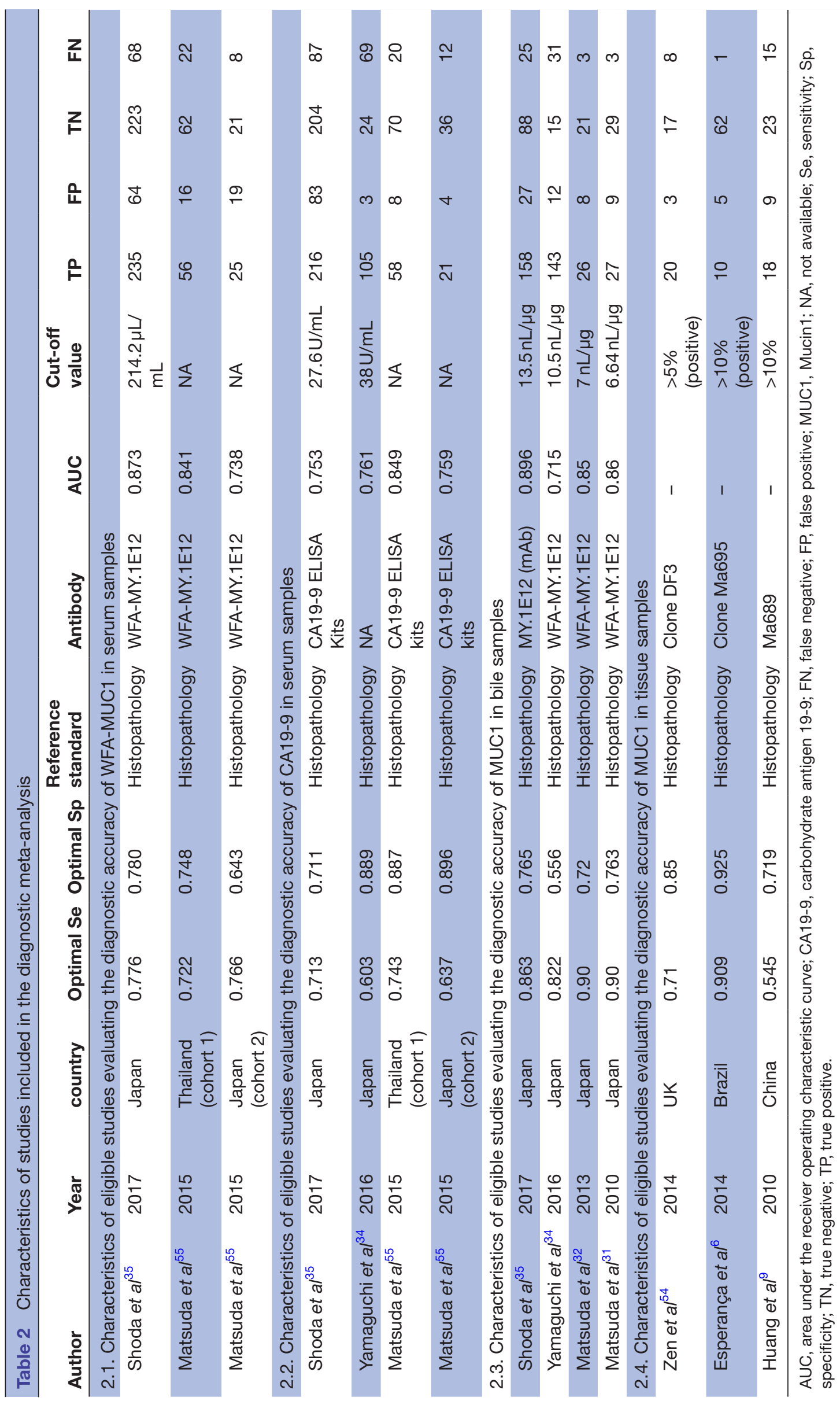


Table 3 Characteristics of eligible studies included in prognostic meta-analysis

\begin{tabular}{|c|c|c|c|c|c|c|c|c|c|}
\hline Author & Year & Country & $\begin{array}{l}\text { Type of } \\
\text { CCA }\end{array}$ & No. of patients & Anti-MUC1 & $\begin{array}{l}\text { Cut-off } \\
\text { (positive/high } \\
\text { expression) }\end{array}$ & $\begin{array}{l}\text { Follow-up } \\
\text { (months) }\end{array}$ & $\begin{array}{l}\text { HR for overall } \\
\text { survival } \\
(95 \% \mathrm{Cl})\end{array}$ & $P$ value \\
\hline Higashi et al ${ }^{7}$ & 2012 & Japan & M-iCCA & 63 & mAb DF3 & $>5 \%(58)$ & $>50$ & $\begin{array}{l}3.34 \text { ( } 0.43 \text { to } \\
25.8)\end{array}$ & 0.168 \\
\hline Huang et $a l^{9}$ & 2010 & China & iCCA & 33 & Clone Ma689 & $>10 \%(18)$ & $>20$ & $\begin{array}{l}1.89(0.79 \text { to } \\
4.511)^{\star}\end{array}$ & $<0.01$ \\
\hline Park et $a l^{10}$ & 2009 & Korea & CCA & 85 & Clone Ma695 & $>10 \%(56)$ & $>50$ & $\begin{array}{l}1.211 \text { ( } 0.403 \text { to } \\
3.640)\end{array}$ & 0.733 \\
\hline Boonla et al ${ }^{12}$ & 2005 & Thailand & iCCA & 87 & Clone Ma695 & $>25 \%(34)$ & $>15$ & $\begin{array}{l}2.19(1.11 \text { to } \\
4.32)\end{array}$ & 0.026 \\
\hline Shibahara et $a l^{13}$ & 2004 & Japan & M-iCCA & 27 & Mab DF3 & $>5 \%(22)$ & $>50$ & $\begin{array}{l}4.536(0.292 \text { to } \\
70.336)\end{array}$ & 0.2797 \\
\hline Matsumura et a $1^{15}$ & 2002 & Japan & M-iCCA & 50 & mAb HMPV & $>5 \%(38)$ & $>50$ & $\begin{array}{l}4.377(1.517 \text { to } \\
12.629)\end{array}$ & 0.0063 \\
\hline Takao et $a l^{37}$ & 1999 & Japan & $\mathrm{CCA}$ & 67 & Mab DF3 & $>25 \%(47)$ & $>50$ & $\begin{array}{l}2.59 \text { (1.19 to } \\
5.63)\end{array}$ & 0.016 \\
\hline
\end{tabular}

*The data were digitised and extracted from the Kaplan-Meier curve using the software designed by Jayne F Tierney and Matthew R Sydes. CCA, cholangiocarcinoma; iCCA, intrahepatic cholangiocarcinoma; M-iCCA, mass-forming intrahepatic cholangiocarcinoma.

\section{Additional analysis}

Studies conducted by several research groups have concluded that the patients with mass-forming intrahepatic CCA or periductal infiltrating CCA had a worse prognosis than patients with other types of CCA regarding the OS. These types of CCA have higher rates of recurrence after resection. ${ }^{56}$ In our meta-analysis for prognosis, subgroup analysis stratified by the histopathological morphology of CCA was conducted to reduce the inconsistency caused by the type of CCA. We found that the OS for patients with positive expression of MUC1 was significantly shorter than that of MUC1 negative group. The overall pooled HRs=2.20. For subjects with mass-forming intrahepatic CCA, HRs=4.17. In addition, a sensitivity analysis was performed to investigate the stability of the pooled HRs. As shown in online supplementary figure 1, the results of pooled HRs were not affected significantly by each individual study.

Molecular Function (MF), Biological Process (BP) and Reactome Pathways of MUC1 in cancer we searched the GO classification system (http://www.pantherdb.org/) to found the Molecular Function, Biological Process and Reactome Pathways of MUC1 in cancer, the search results was summarised in table 4.

\section{DISCUSSION}

As we all known, serum CA19-9 has been widely used as a tumour marker for CCA. However, its diagnostic accuracy is limited since the serum level of CA19-9 can be strongly influenced by the coexisting inflammatory conditions of the biliary tract and this antigen could not be detected in
Lewis gene negative individuals. ${ }^{16} 18$ The most commonly performed diagnostic method for CCA is biliary cytology, which tests the bile sample from a biliary drainage catheter. However, the sensitivity of biliary cytology is extremely low $(20.7 \% \pm 3.5 \%)$ as reported in published study. ${ }^{58}$ In our meta-analysis of the diagnostic capability of markers for CCA, seven prospective trials ${ }^{12} 31-32343555$ and a retrospective study ${ }^{9}$ were eligible for diagnostic analysis that showed that the diagnostic capability of CA19-9 was inferior to other molecules, such as WFA-MUC1.

In the meta-analysis for diagnosis, the diagnostic value of WFA-MUC1 in serum, bile and biliary duct cancer tissue was evaluated and stratified by subgroups of CCA. Two studies ${ }^{35} 55$ with three trials (studies reported by Matsuda et $a l^{55}$ included two cohorts) assessed the diagnostic accuracy of WFA-MUC1 level in serum, the pooled sensitivity of WFA-MUC1 was 0.76 (95\% CI 0.71 to 0.81$)$. The specificity was 0.72 (95\% CI 0.59 to 0.83$)$ and the AUC of SROC was 0.77 (95\% CI 0.73 to 0.81 ). While in three studies ${ }^{34355}$ with four trials assessing the diagnostic accuracy of CA19-9 level in serum, the pooled sensitivity of CA19-9 was 0.67 (95\% CI 0.61 to 0.72 ), the specificity was 0.86 (95\% CI 0.75 to 0.93 ) and the AUC of SROC was 0.75 (95\% CI 0.71 to 0.79 ), which means it would bring a severe error into clinical diagnosis.

The diagnostic capability of serum WFA-MUC1 was superior to that of CA19-9 (as the data showed, AUC WFA-MUC1 $_{\text {vs }}$ $\mathrm{AUC}_{\mathrm{CA19-9}}: 0.77$ (95\% CI 0.73 to 0.81$)$ vs 0.75 (95\% CI 0.71 to 0.79$))$. The sensitivity rate of WFA-MUC1 was higher than that of CA19-9 ( $(95 \%$ CI 0.76 (0.71 to 0.81$)$ vs 0.67 (95\% CI 0.61 to 0.72$))$; nevertheless, the specificity rate 

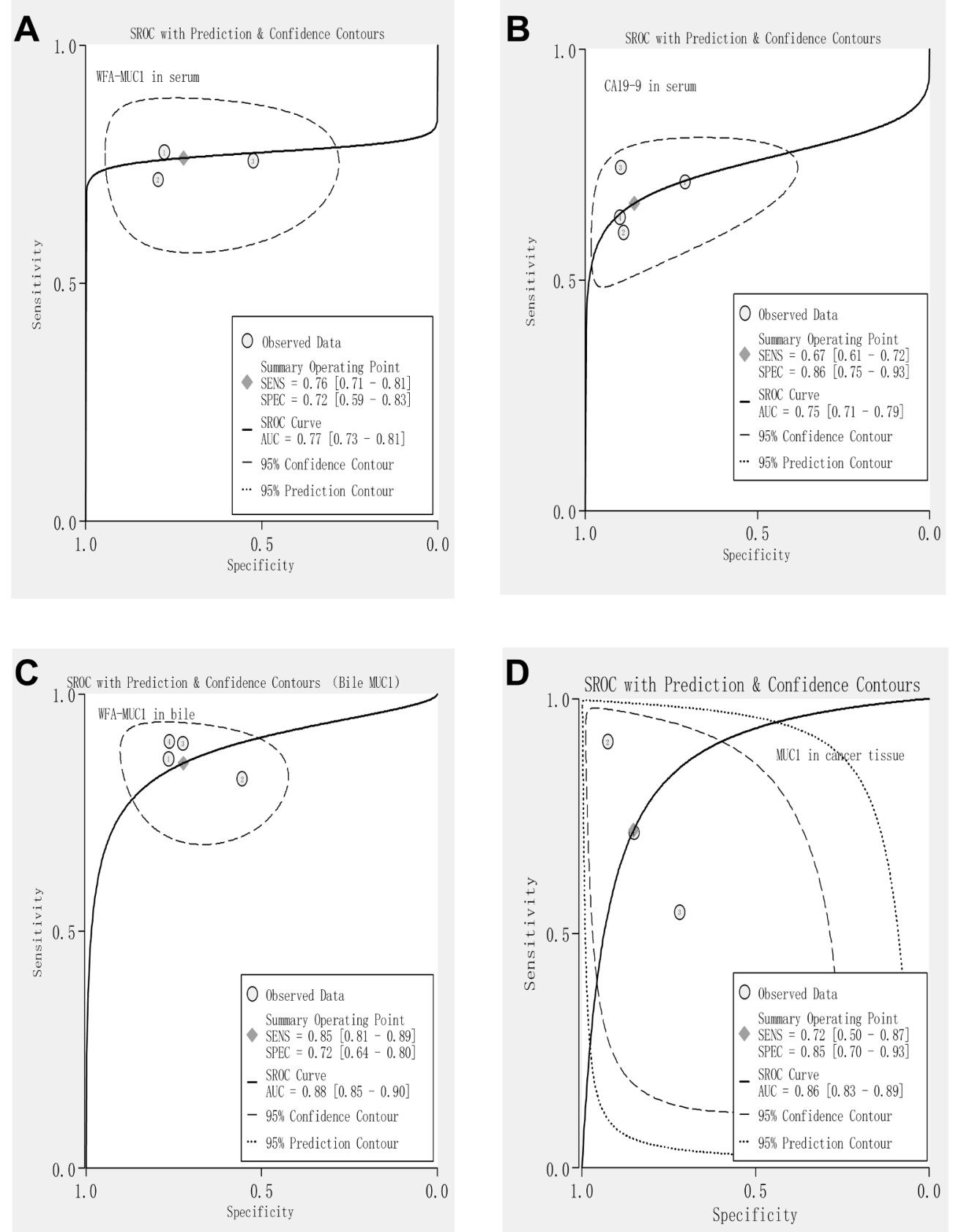

Figure 2 Summary receiver operating characteristic curve (SROC) for WFA-MUC1 and CA19-9. (A) SROC of serum level of MUC1. (B) SROC of serum level of CA19-9. (C) SROC of biliary level of MUC1. (D) SROC of MUC1 in biliary duct cancer tissue. AUC, area under the curve; MUC1, Mucin1; CA19-9, carbohydrate antigen 19-9; WFA-MUC1, Wisteria floribunda agglutinin sialylated-Mucin1.

of serum WFA-MUC1 was less than that of CA19-9 ( $(0.72$ ( $95 \%$ CI 0.59 to 0.83$)$ vs $0.86(95 \%$ CI 0.75 to 0.93$)$ ). In order to discriminate CCA from benign biliary disease, the combination of these two biomarkers may be applied to improve the diagnostic capability of WFA-MUC1 or CA19-9, as reported by previously published trials.

In the four prospective studies 323435 with 343 CAA and 73 gall bladder carcinomas and 209 benign biliary diseases, the diagnostic accuracy of WFA-MUC1 in bile was also assessed. The pooled sensitivity of WFA-MUC1 testing was 0.85 (95\% CI 0.81 to 0.89 ) and specificity was
$0.72(95 \%$ CI 0.64 to 0.80$)$ and AUC of SROC was 0.86 (95\% CI 0.83 to 0.89 ). The diagnostic capability of bile WFA-MUC1 was better than that of serum WFA-MUC1 $\left(\mathrm{AUC}_{\mathrm{MUC1}}\right.$ in bile $\mathrm{Vs} \mathrm{AUC}_{\text {MUC1 in serum }}: 0.86$ (95\% CI 0.83 to 0.89$)$ vs 0.77 ( $95 \%$ CI 0.73 to 0.81$)$ ), which is consistent with the concept that for most of diseases, the diagnostic molecule levels are different between locally and systemically.

As described above, the level of WFA-MUC1 has significantly higher diagnosis accuracy than CA19-9. Furthermore, the diagnostic accuracy of biliary WFA-MUC1 level was better than that in serum. Therefore, the diagnostic 


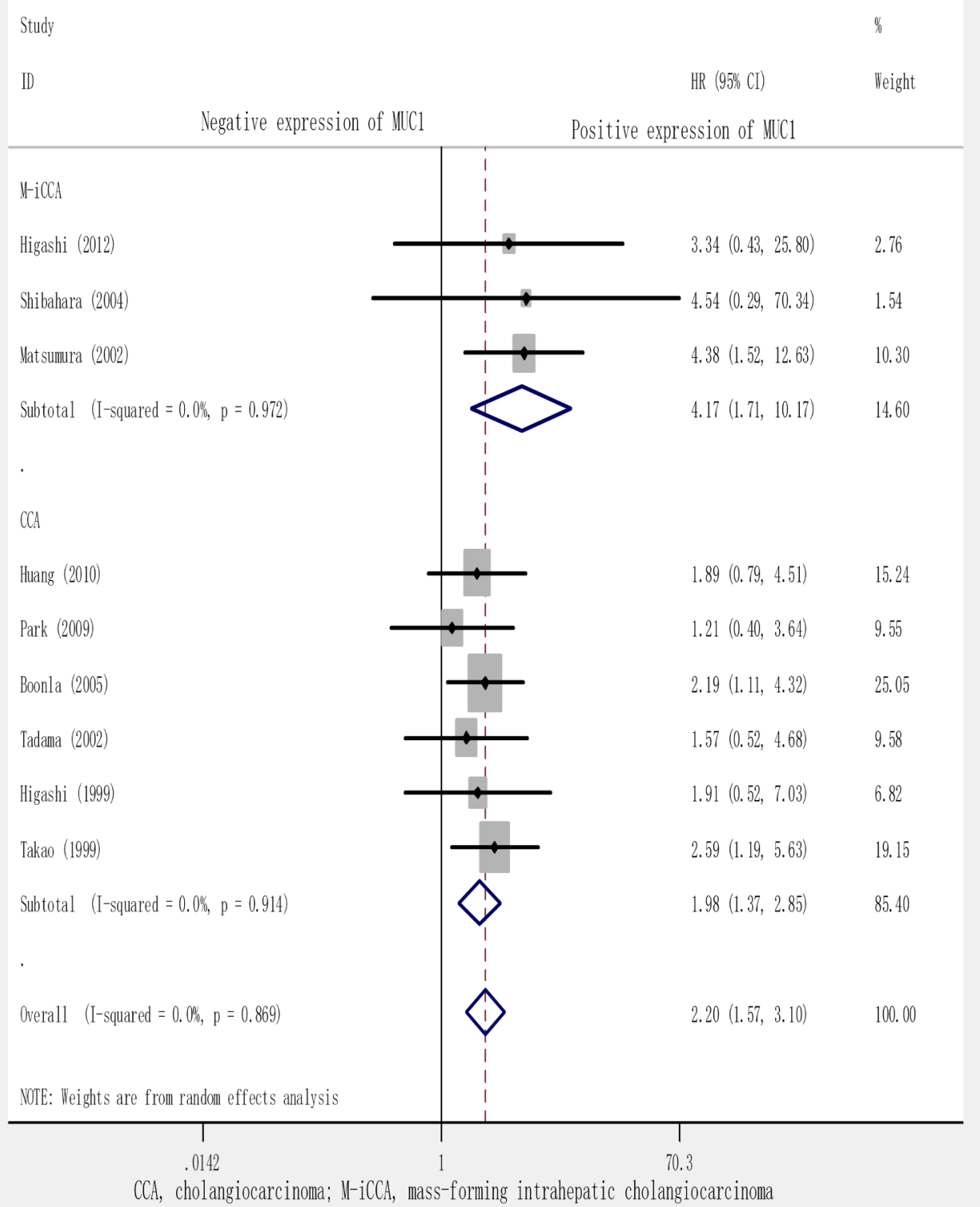

Figure 3 Forest plot of HR evaluating overall survival of patients with resectable CCA. MUC1, Mucin1.

capability of the combined serum CA19-9 and biliary WFA-MUC1 would be better than that of the combination of serum CA19-9 and serum WFA-MUC1 level in discriminating CCA from the benign biliary disease. Such combined measurement would represent a superior diagnostic test for the detection of CCA in daily clinical practice. Unfortunately, as one study ${ }^{55}$ included in the diagnostic meta-analyses did not provide the detailed cut-off value of serum WFA-MUC1 level, nor CA19-9 level, the optimal cut-off value of SROC cannot be estimated by this meta-analysis.

It has been demonstrated that MUC1 expression in various human tumours is related to invasive tumour progression and a poor patient outcome..$^{10365960} \mathrm{In}$ the prognostic meta-analysis, pooled analysis of nine retro-

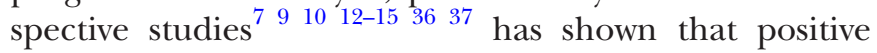
MUC1 expressed of tissue was a poor prognosis factor for resectable CCA (the pooled HRs was 2.20, 95\% CI 1.57 to 3.01), especially for patients with mass-forming intrahepatic CCA (the pooled HRs was $4.17,95 \%$ CI 1.71 to 10.17), which was demonstrated by the subgroup analysis stratified by the morphology of CCA.

It has been reported by publications that around $50 \%-60 \%$ of CCA are identified as perihilar CCA, up to $20 \%$ of CCA are distal, $5 \%$ of tumours are multifocal and up to $20 \%$ of all CCA are intrahepatic. ${ }^{561}$ Different type of CCA demonstrates various epidemiological, morphological and clinical features. A previous meta-analysis ${ }^{43}$ 


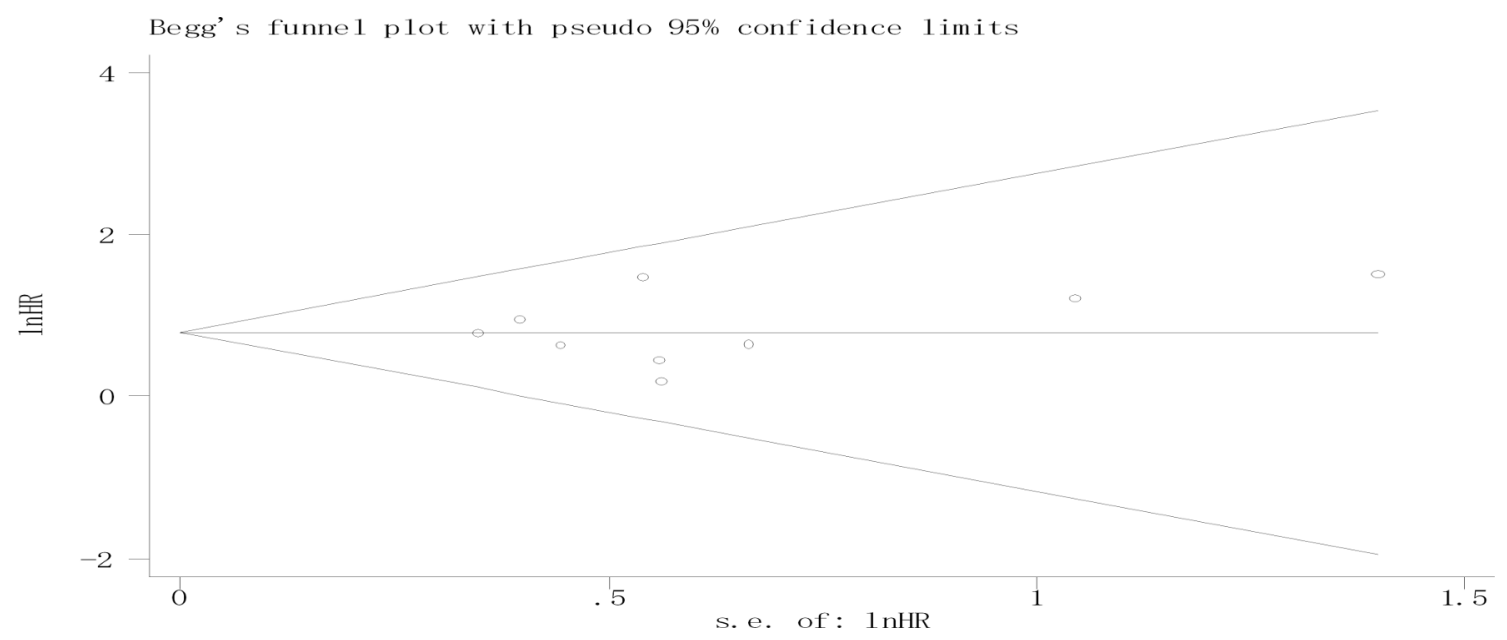

Figure 4 Funnel plot for overall survival.

identified several prognostic biomarkers (EGFR, MUC1, MUC4 and p27) for resectable CCA, with a small number of subjects in the subgroup of evaluating the prognostic role of MUC1 (four studies including 265 subjects with resectable CCA were included in the analysis evaluating the prognostic value of MUC1 expression in tissue). The sample size of the prognostic meta-analysis in our study was doubled (9 studies including 511 patients with resectable CCA), and our study provided more explicit description and analysis. Subgroup analysis and sensitivity analysis were conducted to get more reliable results. The pooled HRs result in our study showed that overexpression of MUC1 in tissue was a poor prognostic index for resectable CCA, in particular for patients with massforming intrahepatic CCA.

Predictive biomarkers could serve as the key point for personalised cancer treatments such as verifying the chemosensitivity of CCA and developing vaccines to CCA. Up to now, VEGFR, EGFR, HER2, MEK and BRAF have been the focus for the studies evaluating molecular targeting therapies for CCA. ${ }^{62}$ Along with the better understanding of the pathogenesis of CCA mediated by
MUC1, MUC1 may become a new focus of targeted therapies for CCA.

\section{The strength and limitation of this study}

This meta-analysis, to our best knowledge, is the first study to evaluate the diagnostic value of WFA-MUC1 and prognostic role of MUC1 for human CCA; we obtained data about the prognostic and/or diagnostic capability of WFA-MUC1/MUC1 for CCA from16 trials, which were identified by systematically searching four databases; all subjects with CCA included in this study were diagnosed by pathologist postoperatively. To avoid the possible bias brought by including studies only with reported HRs that may affect the significance of the statistical analysis, we digitised and extracted the HR data from Kaplan-Meier curves in three studies. ${ }^{9}{ }^{146}$ In addition, sensitivity analysis and subgroup analysis, which was stratified by the morphology of CCA, made our results of the pooled HRs more stable. To analyse the diagnostic capability of WFA-MUC1, we separately assessed the diagnostic accuracy of WFA-MUC1 level in serum, in bile and in tissue. A comparison of diagnostic accuracy between WFA-MUC1

Table 4 The molecular function (MF), biological process (BP) and reactome pathways of MUC1 in cancer

Gene Ontology MF p53 binding, transcription coregulator activity, protein binding and RNA polymerase II proximal Complete promoter sequence-specific DNA binding.

Gene Ontology BP DNA damage response, signal transduction by p53 class mediator resulting in transcription of p21 class complete mediator, negative regulation of cell adhesion mediated by integrin, positive regulation of transcription from RNA polymerase II promoter in response to stress, DNA damage response, signal transduction by p53 class mediator resulting in cell cycle arrest, negative regulation of transcription by competitive promoter binding, regulation of transcription from RNA polymerase II promoter in response to stress, cytokine-mediated signalling pathway, negative regulation of intrinsic apoptotic signalling pathway in response to DNA damage by p53 class mediator, O-glycan processing, positive regulation of histone H4 acetylation and stimulatory C-type lectin receptor signalling pathway

Reactome pathways O-linked glycosylation of mucins, Metabolism of proteins, O-linked glycosylation, Defective C1GALT1C1 causes Tn polyagglutination syndrome, diseases of glycosylation, termination of O-glycan biosynthesis, defective GALNT3 causes familial hyperphosphatemic tumorous calcinosis, defective GALNT12 causes colorectal cancer 1, post-translational protein modification, disease, diseases associated with O-glycosylation of proteins. 
level and CA19-9 level in serum, as well as a comparison of diagnostic accuracy between the WFA-MUC1 level in serum and in bile were also conducted in our study, as previously clinical trials described.

While our present study could provide a great amount of useful information, limitations of our study should be kept in mind. First, majority of the subjects included in this meta-analysis were from Asian hospitals (data on prognostic meta-analysis were retrieved from Japan, China, Korea and Thailand; data on diagnostic meta-analysis were from Japan, Thailand, China, Brazil and the UK). There may be biological differences in terms of tumour behaviours among populations from different regions worldwide. The phenomenon has been reported on the mortality of stomach cancer between eastern countries and western countries. ${ }^{63}$ Second, four different cut-off values of positive MUC1 immunostaining ( $>5 \%$ of carcinoma cells stained was defined as the cut-off point by four studies, $>10 \%$ defined by two studies, $>20 \%$ identified by one study and $>25 \%$ defined by another two studies) and four different MUC1 antibodies (mAb DF3, Clone Ma689, Clone Ma695 and mAb HMPV) were used in the nine included studies in our prognostic meta-analysis. Lack of consistency on cut-off value and the type of MUC1 antibody used resulted in considerable heterogeneity. Third, in the diagnostic meta-analysis, although majority of subjects in the biliary tract carcinoma group were diagnosed with CCA, a total of 73 subjects with gall bladder carcinomas were included in this group to evaluate the diagnostic capability of biliary level of MUC1 and serum level of CA19-9. Fiftynine patients with gall bladder carcinomas were included in the evaluation of the diagnostic capability of serum level of WFA-MUC1. The heterogeneity caused by the inconsistency of participants cannot be underestimated since WFA-MUC1 can serve as an independent predictor of hepatocellular carcinoma recurrence. ${ }^{64}$ It may be useful for discriminating gall bladder carcinoma from benign gall bladder disease. Fourth, given that only seven trials with a small number of patients were eligible for the diagnostic meta-analysis and two of them did not provide the cut-off value of WFA-MUC1 and CA19-9 in serum, we cannot give an estimated optimal cut-off value for WFA-MUC1 level in serum. Finally, all data in our study was retrieved from subjects with resectable CCA or gall bladder carcinoma, there may be some difference in the pathogenesis between resectable and unresectable CCA.

\section{CONCLUSIONS}

This paper highlighted the importance of WFA-MUC1. It has a better diagnostic capability than CA19-9, and the diagnostic capability of the biliary level of WFA-MUC1 was superior to that in the serum. Furthermore, MUC1 could served as a prognosis factor for poor outcomes of resectable CCA, particularly in mass-forming intrahepatic CCA.

Larger, multicentre studies are still needed for better understanding of the molecular pathogenesis of CCA, developing combined kits to conveniently test the serum/biliarylevel of MUC1 and serum level of CA19-9 in routine clinical practice, providing an optimal cut-off value of WFA-MUC1 with higher diagnostic accuracy for CCA and benefiting the populations from different regions worldwide.

Contributors ZT: study concept and design; development of methodology, collection and extraction of data, statistical analysis and interpretation of data and drafting of the manuscript; and critical revision of the manuscript for significant intellectual content. YY and XW: development of methodology, collection and extraction of data and statistical analysis. WM and XL: funding application and study supervision

Funding This study was supported by the West Light Foundation of the Chinese Academy of Science (2015(90)).

Disclaimer The funding sources played no roles in the study design, experiment method development, data collection, organization, analysis and interpretation, nor in the manuscript drafting, reviewing or approval.

Competing interests None declared.

Patient consent Not required.

Ethics approval This study did not require ethical approval as the data used have been published previously, and hence are already in the public domain.

Provenance and peer review Not commissioned; externally peer reviewed.

Data sharing statement Extracted data are available upon request to the corresponding author.

Open access This is an open access article distributed in accordance with the Creative Commons Attribution Non Commercial (CC BY-NC 4.0) license, which permits others to distribute, remix, adapt, build upon this work non-commercially, and license their derivative works on different terms, provided the original work is properly cited, appropriate credit is given, any changes made indicated, and the use is non-commercial. See: http://creativecommons.org/licenses/by-nc/4.0/.

\section{REFERENCES}

1. Razumilava N, Gores GJ. Cholangiocarcinoma. The Lancet 2014;383:2168-79.

2. Eckel F, Schmid RM. Chemotherapy in advanced biliary tract carcinoma: a pooled analysis of clinical trials. Br J Cancer 2007;96:896-902.

3. Park J, Kim MH, Kim KP, et al. Natural history and prognostic factors of advanced cholangiocarcinoma without surgery, chemotherapy, or radiotherapy: A large-scale observational study. Gut Liver 2009;3:298-305.

4. Tang Z, Yang Y, Zhao Z, et al. The clinicopathological factors associated with prognosis of patients with resectable perihilar cholangiocarcinoma: A systematic review and meta-analysis. Medicine 2018;97:e11999.

5. Khan SA, Davidson BR, Goldin RD, et al. Guidelines for the diagnosis and treatment of cholangiocarcinoma: an update. Gut 2012;61:1657-69.

6. Esperança ABT, Camacho AHdaS, Monteiro JBM, et al. Mucins and NCAM (CD56) in intrahepatic cholangiocarcinogenesis. J Bras Patol Med Lab 2014;50:216-20.

7. Higashi M, Yamada N, Yokoyama S, et al. Pathobiological implications of MUC16/CA125 expression in intrahepatic cholangiocarcinoma-mass forming type. Pathobiology 2012;79:101-6.

8. Silsirivanit A, Araki N, Wongkham C, et al. A novel serum carbohydrate marker on mucin 5AC: values for diagnostic and prognostic indicators for cholangiocarcinoma. Cancer 2011;117:3393-403.

9. Huang F, Zhou QB, Chen RF. Expression and significance of MUC1 in hepatolithiasis associated with intrahepatic cholangiocarcinoma. Chinese Archives of General Surgery 2010;4:424-7.

10. Park SY, Roh SJ, Kim YN, et al. Expression of MUC1, MUC2, MUC5AC and MUC6 in cholangiocarcinoma: prognostic impact. Oncol Rep 2009;22:649-57.

11. Matull WR, Andreola F, Loh A, et al. MUC4 and MUC5AC are highly specific tumour-associated mucins in biliary tract cancer. $\mathrm{Br} J$ Cancer 2008;98:1675-81.

12. Boonla C, Sripa B, Thuwajit P, et al. MUC1 and MUC5AC mucin expression in liver fluke-associated intrahepatic cholangiocarcinoma. World J Gastroenterol 2005;11:4939-46.

13. Shibahara H, Tamada S, Higashi M, et al. MUC4 is a novel prognostic factor of intrahepatic cholangiocarcinoma-mass forming type. Hepatology 2004;39:220-9. 
14. Tamada S, Goto M, Nomoto M, et al. Expression of MUC1 and MUC2 mucins in extrahepatic bile duct carcinomas: its relationship with tumor progression and prognosis. Pathol Int 2002;52:713-23.

15. Matsumura N, Yamamoto $M$, Aruga A, et al. Correlation between expression of MUC1 core protein and outcome after surgery in massforming intrahepatic cholangiocarcinoma. Cancer 2002;94:1770-6.

16. Chen CY, Shiesh SC, Tsao HC, et al. The assessment of biliary CA 125, CA 19-9 and CEA in diagnosing cholangiocarcinomathe influence of sampling time and hepatolithiasis. Hepatogastroenterology 2002;49:616-20.

17. Patel AH, Harnois DM, Klee GG, et al. The utility of CA 19-9 in the diagnoses of cholangiocarcinoma in patients without primary sclerosing cholangitis. Am J Gastroenterol 2000;95:204-7.

18. Narimatsu $H$, Iwasaki $H$, Nakayama $F$, et al. Lewis and secretor gene dosages affect CA19-9 and DU-PAN-2 serum levels in normal individuals and colorectal cancer patients. Cancer Res 1998;58:512-8.

19. Xu H, Inagaki $\mathrm{Y}$, Tang $\mathrm{W}$, et al. Elevation of serum $\mathrm{KL}-6$ mucin levels in patients with cholangiocarcinoma. Hepatogastroenterology 2008;55:2000-4.

20. Cheon YK, Cho YD, Moon JH, et al. Diagnostic utility of interleukin-6 (IL-6) for primary bile duct cancer and changes in serum IL-6 levels following photodynamic therapy. Am J Gastroenterol 2007;102:2164-70.

21. Lumachi F, Lo Re G, Tozzoli R, et al. Measurement of serum carcinoembryonic antigen, carbohydrate antigen 19-9, cytokeratin-19 fragment and matrix metalloproteinase-7 for detecting cholangiocarcinoma: a preliminary case-control study. Anticancer Res 2014;34:6663-7.

22. Uenishi T, Yamazaki O, Tanaka H, et al. Serum cytokeratin 19 fragment (CYFRA21-1) as a prognostic factor in intrahepatic cholangiocarcinoma. Ann Surg Oncol 2008;15:583-9.

23. Valle J, Wasan $\mathrm{H}$, Palmer DH, et al. Cisplatin plus gemcitabine versus gemcitabine for biliary tract cancer. N Engl J Med 2010;362:1273-81.

24. Abbas $\mathrm{G}$, Lindor KD. Cholangiocarcinoma in primary sclerosing cholangitis. J Gastrointest Cancer 2009;40(1-2):19-25.

25. Hultcrantz R, Olsson R, Danielsson A, et al. A 3-year prospective study on serum tumor markers used for detecting cholangiocarcinoma in patients with primary sclerosing cholangitis. $J$ Hepatol 1999;30:669-73.

26. Gores GJ. Early detection and treatment of cholangiocarcinoma. Liver Transp/ 2000;6(6 Suppl 2):s30-s34.

27. Hamada E, Taniguchi T, Baba S, et al. Investigation of unexpected serum CA19-9 elevation in Lewis-negative cancer patients. Ann Clin Biochem 2012;49(Pt 3):266-72.

28. Gendler SJ, Lancaster CA, Taylor-Papadimitriou J, et al. Molecular cloning and expression of human tumor-associated polymorphic epithelial mucin. J Biol Chem 1990;265:15286-93.

29. Lan MS, Batra SK, Qi WN, et al. Cloning and sequencing of a human pancreatic tumor mucin cDNA. J Biol Chem 1990;265:15294-9.

30. Brockhausen I. Pathways of O-glycan biosynthesis in cancer cells. Biochim Biophys Acta 1999;1473:67-95.

31. Matsuda A, Kuno A, Kawamoto T, et al. Wisteria floribunda agglutinin-positive mucin 1 is a sensitive biliary marker for human cholangiocarcinoma. Hepatology 2010;52:174-82.

32. Matsuda A, Kuno A, Matsuzaki H, et al. Glycoproteomicsbased cancer marker discovery adopting dual enrichment with Wisteria floribunda agglutinin for high specific glyco-diagnosis of cholangiocarcinoma. J Proteomics 2013;85:1-11.

33. Xu F, Liu F, Zhao H, et al. Prognostic significance of mucin antigen muc1 in various human epithelial cancers: A meta-analysis. Medicine 2015;94:e2286

34. Yamaguchi T, Yokoyama Y, Ebata T, et al. Verification of WFASialylated MUC1 as a sensitive biliary biomarker for human biliary tract cancer. Ann Surg Oncol 2016;23:671-7.

35. Shoda J, Matsuda A, Shida T, et al. Wisteria floribunda agglutininsialylated mucin core polypeptide 1 is a sensitive biomarker for biliary tract carcinoma and intrahepatic cholangiocarcinoma: a multicenter study. J Gastroenterol 2017;52:218-28.

36. Higashi M, Yonezawa S, Ho JJ, Jj H, et al. Expression of MUC1 and MUC2 mucin antigens in intrahepatic bile duct tumors: its relationship with a new morphological classification of cholangiocarcinoma. Hepatology 1999;30:1347-55.

37. Takao S, Uchikura K, Yonezawa S, et al. Mucin core protein expression in extrahepatic bile duct carcinoma is associated with metastases to the liver and poor prognosis. Cancer 1999;86:1966-75.

38. Tierney JF, Stewart LA, Ghersi D, et al. Practical methods for incorporating summary time-to-event data into meta-analysis. Trials 2007;8:16.
39. Kelsey TW, Anderson RA, Wright P, et al. Data-driven assessment of the human ovarian reserve. Mol Hum Reprod 2012;18:79-87.

40. Iliodromiti S, Kelsey TW, Anderson RA, et al. Can anti-Mullerian hormone predict the diagnosis of polycystic ovary syndrome? A systematic review and meta-analysis of extracted data. J Clin Endocrinol Metab 2013;98:3332-40.

41. Ciaccio AD. Bootstrap and Nonparametric Predictors to Impute Missing Data, 2011.

42. Rahbari NN, Aigner M, Thorlund K, et al. Meta-analysis shows that detection of circulating tumor cells indicates poor prognosis in patients with colorectal cancer. Gastroenterology 2010;138:1714-26.

43. Ruys AT, Groot Koerkamp B, Wiggers JK, et al. Prognostic biomarkers in patients with resected cholangiocarcinoma: a systematic review and meta-analysis. Ann Surg Oncol 2014;21:487-500.

44. Higgins JP, Green S. Cochrane handbook for systematic reviews of interventions [internet]. Naunyn-Schmiedebergs Archiv für experimentelle Pathologie und Pharmakologie 2011;2009:S38.

45. Whiting P, Rutjes AW, Reitsma JB, et al. The development of QUADAS: a tool for the quality assessment of studies of diagnostic accuracy included in systematic reviews. BMC Med Res Methodol 2003;3:25.

46. van Rheenen PF, Van de Vijver E, Fidler V. Faecal calprotectin for screening of patients with suspected inflammatory bowel disease: diagnostic meta-analysis. BMJ 2010;341:c3369.

47. Whiting P, Harbord R, Kleijnen J. No role for quality scores in systematic reviews of diagnostic accuracy studies. BMC Med Res Methodol 2005:5:19.

48. Stroup DF, Berlin JA, Morton SC, et al. Meta-analysis of observational studies in epidemiology: a proposal for reporting. Meta-analysis Of Observational Studies in Epidemiology (MOOSE) group. JAMA 2000;283:2008-12.

49. DerSimonian R, Laird N. Meta-analysis in clinical trials revisited. Contemp Clin Trials 2015;45-139-45.

50. Xie S, Wang K, Xu H, et al. PRISMA-Extracapsular dissection versus superficial parotidectomy in treatment of benign parotid tumors: evidence from 3194 patients. Medicine 2015;94:e1237.

51. Harbord RM, Deeks JJ, Egger M, et al. A unification of models for meta-analysis of diagnostic accuracy studies. Biostatistics 2007;8:239-51.

52. Higgins JP, Thompson SG, Deeks JJ, et al. Measuring inconsistency in meta-analyses. BMJ 2003;327:557-60.

53. Egger M, Davey Smith G, Schneider M, et al. Bias in meta-analysis detected by a simple, graphical test. BMJ 1997;315:629-34.

54. Zen Y, Britton D, Mitra V, et al. Tubulin $\beta$-III: a novel immunohistochemical marker for intrahepatic peripheral cholangiocarcinoma. Histopathology 2014;65:784-92.

55. Matsuda A, Kuno A, Nakagawa T, et al. Lectin microarray-based sero-biomarker verification targeting aberrant O-linked glycosylation on mucin 1. Anal Chem 2015;87:7274-81.

56. Yamamoto Y, Shimada K, Sakamoto Y, et al. Clinicopathological characteristics of intrahepatic cholangiocellular carcinoma presenting intrahepatic bile duct growth. J Surg Oncol 2009;99:161-5.

57. Shimada K, Sano T, Sakamoto Y, et al. Surgical outcomes of the mass-forming plus periductal infiltrating types of intrahepatic cholangiocarcinoma: a comparative study with the typical massforming type of intrahepatic cholangiocarcinoma. World J Surg 2007;31:2016-22.

58. Tsuchiya T, Yokoyama Y, Ebata T, et al. Randomized controlled trial on timing and number of sampling for bile aspiration cytology. $J$ Hepatobiliary Pancreat Sci 2014;21:433-8.

59. Yonezawa S, Goto M, Yamada N, et al. Expression profiles of MUC1, MUC2, and MUC4 mucins in human neoplasms and their relationship with biological behavior. Proteomics 2008;8:3329-41.

60. Utsunomiya T, Yonezawa S, Sakamoto $\mathrm{H}$, et al. Expression of MUC1 and MUC2 mucins in gastric carcinomas: its relationship with the prognosis of the patients. Clin Cancer Res 1998;4:2605-14.

61. Khan SA, Emadossadaty S, Ladep NG, et al. Rising trends in cholangiocarcinoma: is the ICD classification system misleading us? $J$ Hepatol 2012;56:848-54.

62. Zhu AX, Hezel AF. Development of molecularly targeted therapies in biliary tract cancers: reassessing the challenges and opportunities. Hepatology 2011;53:695-704.

63. Strong VE, Song KY, Park $\mathrm{CH}$, et al. Comparison of gastric cancer survival following RO resection in the United States and Korea using an internationally validated nomogram. Ann Surg 2010;251:640-6.

64. Tamaki N, Kuno A, Matsuda A, et al. Serum wisteria floribunda agglutinin-positive sialylated mucin 1 as a marker of progenitor/ biliary features in hepatocellular carcinoma. Sci Rep 2017;7:244. 